\title{
Band-to-Mott-insulator transformations in four-component alkali-metal fermions at half-filling
}

\author{
J. Jaramillo, S. Greschner, and T. Vekua \\ Institut für Theoretische Physik, Leibniz Universität Hannover, D-30167 Hannover, Germany
}

(Received 14 May 2013; published 11 October 2013)

\begin{abstract}
Under the influence of an external magnetic field and spin-changing collisions, the band insulator state of one-dimensional $s$-wave repulsively interacting four-component fermions at half-filling transforms into Mott insulator states with a spontaneously doubled unit cell: a dimerized state for shallow lattices and a Néel state for deep lattices via an intermediate topological state. These Mott insulator phases could be of special interest for experiments as they can be reached starting from band insulator state and changing magnetic field adiabatically.
\end{abstract}

DOI: $10.1103 /$ PhysRevA.88.043616

PACS number(s): 67.85.-d, 03.75.Mn, 05.30.Fk, 71.10.Fd

One of the outstanding open questions in condensed matter theory is whether the two-dimensional (2D) Hubbard model of electrons is the minimal model that captures the behavior of high- $T_{c}$ superconductors. In reduced spatial dimensions enhanced quantum and thermal fluctuations often invalidate mean-field-like treatments. In 2D, a rigorous analytical approach, like one-dimensional (1D) Bethe ansatz, does not exist and there is limited support from unbiased large-scale numerical simulations because of the notorious negative sign problem of Monte Carlo calculations. Ultracold spinor fermions in optical lattices present an extraordinary tool for simulating the 2D Hubbard model under well-controlled conditions [1,2]. The metal-to-Mott-insulator (MI) transition was experimentally observed with two-component Fermi gases $[3,4]$. At sufficiently low temperatures the MI phase should acquire a magnetic Néel (antiferromagnetic) ordering. Still it has not been resolved [5]; the main obstacle is the absence of efficient cooling methods in the presence of an optical lattice [6].

By increasing the number of components above two, an interesting possibility of hosting exotic ground states like 2D spin liquids $[7,8]$ or 1D topological states $[9,10]$ emerges. A novel ingredient in multicomponent alkali-metal gases, different from the two-component case, is the presence of spin-changing collisions: two interacting atoms cannot only exchange their initial internal hyperfine states, but they can also change them to new values. The spin-changing collisions open a fascinating prospect to arrive at unconventional MI ground states of multicomponent Fermi gases starting from the band insulator (BI) state made of only two components having vanishing entropy per particle. For fermions due to the Pauli principle the minimal number of components required for BI to MI transformation is four.

In this work we present the ground-state phases of fourcomponent alkali-metal fermions at half-filling obtained by nonperturbative analytical and numerical tools, particularly tailored for studying 1D systems. We identify various MI phases: Dimer and Néel states spontaneously break discrete lattice translational symmetry and are characterized by doubly degenerate ground state in thermodynamic limit and local order parameters; whereas singlet and a topological Haldane phase do not break any microscopic symmetry and are characterized by unique ground state and nonlocal parity and string orders, respectively. Even though these different MI phases are manifested below the ultralow temperatures, typical to the superexchange scale, the fact that one can start from the BI state and by adiabatically changing the magnetic field enter into these nontrivial states, makes the system of half-filled four-component alkali-metal fermions a particularly attractive candidate to resolve ground-state spin order in experiments on ultracold lattice gases. Note that the spin-changing collision processes, crucial for BI to MI transformations, are maximally pronounced at half-filling.

Our main result, the ground-state phase diagram of four components of ${ }^{40} \mathrm{~K}$ atoms, is presented in Fig. 1. Most importantly all states can be explored just by changing the lattice depth and strength of the external magnetic field without the need to modify the natural values of scattering lengths. This phase diagram is not particular of ${ }^{40} \mathrm{~K}$ atoms; it captures generic phases of repulsive four-component alkalimetal fermions at half-filling, provided these components do not form complete hyperfine multiplet. For the case of hyperfine spin $f=3 / 2$, due to the unusually large internal symmetry [12] holding even for finite quadratic Zeeman coupling [13], only dimer and singlet MI phases are realized as depicted in Fig. 3(b). Singlet phases are connected to the BI state with crossover behavior.

$f=3 / 2$ multiplet. We start from the case of a hyperfine spin $f=3 / 2$ [14]. The lattice Hamiltonian that describes $s$ wave interacting fermions in the presence of quadratic Zeeman coupling $q$ is given by

$$
\begin{aligned}
H & =\sum_{j=1}^{L} H_{0, j}+\sum_{j=1}^{L} H_{\mathrm{int}, j}, \\
H_{0, j} & =\sum_{\alpha=-3 / 2}^{3 / 2}\left[-t\left(\psi_{\alpha, j}^{\dagger} \psi_{\alpha, j+1}+\text { H.c. }\right)-q \alpha^{2} n_{\alpha, j}\right], \\
H_{\mathrm{int}, j} & =\sum_{F,\left|m_{F}\right| \leqslant F} g_{F} P_{F, m_{F}}^{\dagger}(j) P_{F, m_{F}}(j) .
\end{aligned}
$$

The operator $\psi_{\alpha}$ annihilates an atom in the hyperfine spin state $\left|f=3 / 2, m_{f}=\alpha\right\rangle$ and $n_{\alpha, j}=\psi_{\alpha, j}^{\dagger} \psi_{\alpha, j}$. Interaction coefficients $g_{F}$ are proportional to scattering strengths $\sim 4 \pi \hbar^{2} a_{F} / m$ [15], where $m$ is the atomic mass, $a_{F}$ are the $s$-wave scattering lengths, and $P_{F, m_{F}}^{\dagger}(j)=$ $\sum_{\alpha, \beta}\left\langle F, m_{F} \mid \alpha \beta\right\rangle \psi_{\alpha, j}^{\dagger} \psi_{\beta, j}^{\dagger}$ create on-site pairs with total spin $F$ and projection $m_{F}$.

Due to the Pauli principle only channels with $F=0,2$ are allowed in the low-energy scattering. Interactions are assumed 


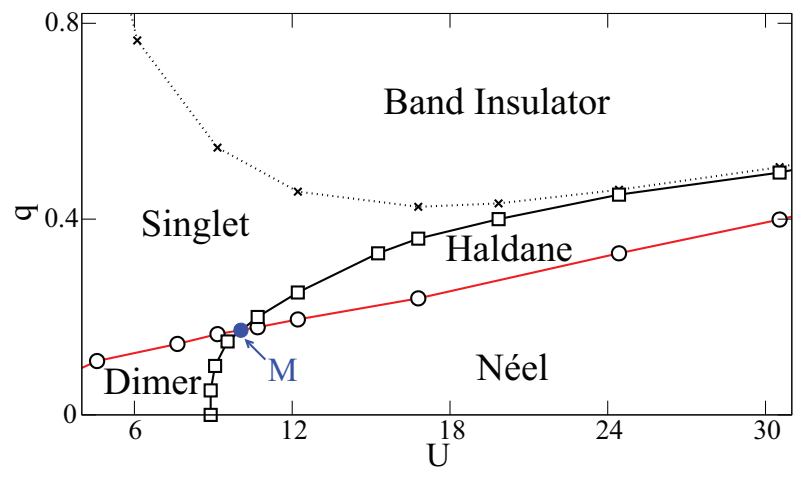

FIG. 1. (Color online) Numerical ground-state phase diagram for a four-component mixture of ${ }^{40} K$ atoms at half-filling with average interaction energy $U \sim \frac{4 \pi \hbar^{2}}{m}\left(a_{0}^{K}+a_{2}^{K}+a_{4}^{K}+a_{6}^{K}+a_{8}^{K}\right) / 5$ $\left(a_{0}^{K}, \ldots a_{8}^{K}\right.$ being the $s$-wave scattering lengths of the lowest hyperfine ground-state manifold $f=9 / 2$ of ${ }^{40} K$ ) and quadratic Zeeman coupling $q$ both in units of hopping $t$ [11]. Dotted line indicates sharp crossover between $\mathrm{BI}$ and $\mathrm{MI}$ and continuous lines are quantum phase transition lines, crossing at multicritical point $\mathrm{M}$.

to be repulsive and we concentrate on the case of two fermions per lattice site on average and zero net magnetization $N_{\alpha}=$ $N_{-\alpha}$, where $N_{\alpha}=\sum_{j}\left\langle n_{\alpha, j}\right\rangle$.

Usually, in alkali-metal atoms, differences between the $s$-wave scattering lengths are a few percent of the average scattering length, hence deviations from $S U$ (4) symmetry $\left(a_{0}=a_{2}\right.$ case) are small. In the vicinity of the $S U(4)$ point, without magnetic field, a unique gapped phase with spontaneously doubled lattice constant and dimer order (spin Peierls) is realized [16,17]; it has an order parameter $D=$ $\left|\sum_{\alpha, j}(-1)^{j}\left\langle\psi_{\alpha, j}^{\dagger} \psi_{\alpha, j+1}\right\rangle\right| /(L-1)>0$. We show that for $a_{0} \neq a_{2}$ the magnetic field above a critical value restores translational symmetry via quantum phase transition belonging to the second-order Ising universality class and the system enters a site-singlet state that is adiabatically connected to the BI of the two-component Fermi Hubbard model. For the case $a_{0}=a_{2}$, as in alkali-metal earth atoms [18], the dimer state is separated from BI by a gapless Luttinger liquid state.

For the two-component repulsive Hubbard model at half-filling the charge sector is gapped and the low-energy effective theory is described by the spin sector. Similarly, in the case of four-component fermions at half-filling, the charge sector is gapped and instead of one spin sector there are three flavor sectors described by the set of dual bosonic fields $\phi_{\eta}$ and $\theta_{\eta} \quad\left(\eta=v, t_{1}, t_{2}\right), \partial_{x} \phi_{v} \sim n_{\frac{3}{2}, j}+n_{-\frac{3}{2}, j}-n_{\frac{1}{2}, j}-n_{-\frac{1}{2}, j}$, $\partial_{x} \phi_{t_{1}, t_{2}} \sim n_{\frac{3}{2}, j}-n_{-\frac{3}{2}, j} \mp n_{\frac{1}{2}, j} \pm n_{-\frac{1}{2}, j}$ with $\left[\theta_{\eta}(x), \partial_{y} \phi_{\eta^{\prime}}\right]=$ $i \delta_{\eta, \eta^{\prime}} \delta(x-y)$. All flavor sectors are generically gapped [19]. The quadratic Zeeman effect couples only to the chiral sector $\phi_{v}$. Chirality is defined as $\tau=\frac{1}{2 L} \sum_{j}\left\langle\left[n_{\frac{3}{2}, j}+n_{-\frac{3}{2}, j}-n_{\frac{1}{2}, j}-n_{-\frac{1}{2}, j}\right]\right\rangle ; \quad$ it measures imbalance between $\pm \frac{1}{2}$ and $\pm \frac{3}{2}$ components. With relevant $4 k_{F}$ Umklapp processes, the important low-energy sector is governed by the following Hamiltonian density,

$$
\begin{aligned}
\mathcal{H}_{v}= & \frac{v_{v}}{2}\left\{K_{v}\left(\partial_{x} \theta_{v}\right)^{2}+\frac{1}{K_{v}}\left(\partial_{x} \phi_{v}\right)^{2}\right\} \\
& -g \cos \sqrt{4 \pi} \phi_{v}-g_{\gamma} \cos \sqrt{4 \pi} \theta_{v}-\frac{2 q}{\sqrt{\pi}} \partial_{x} \phi_{v},
\end{aligned}
$$

where $\quad v_{v}=\sqrt{v_{F}^{2}+\left(g_{0}-3 g_{2}\right) v_{F} / 2 \pi}, \quad K_{v}=$ $\sqrt{2 \pi v_{F} /\left(2 \pi v_{F}+g_{0}-3 g_{2}\right)}, \quad v_{F}$ being the Fermi velocity of free fermions. Coupling constants $g \sim g_{0}+g_{2}$ and $g_{\gamma} \sim g_{2}-g_{0}$, include proportionality coefficients involving ultraviolet cutoff and averages of massive charge and $\phi_{t_{1,2}}$ fields which are governed by bosonic Hamiltonians of the sine-Gordon type [19].

Setting $v_{v}=K_{v}=1$ the effective model in Eq. (2) is diagonalized by the refermionization procedure [20] and Bogoliubov transformation. The low-energy excitation spectrum allows us to locate quantum phase transition and determine its nature. In general for $g_{\gamma} \neq 0$ the excitation spectrum is gapped, except at a critical magnetic field $q=q_{c}$. The lowenergy spectrum is given by two fermionic branches, $\omega_{ \pm}(k)=$ $\sqrt{g^{2}+g_{\gamma}^{2}+4 q^{2}+k^{2} \pm 2 \sqrt{g^{2} g_{\gamma}^{2}+4 q^{2}\left(g^{2}+k^{2}\right)}}$. Only one branch becomes gapless for $q_{c}=\sqrt{g^{2}-g_{\gamma}^{2}} / 2, \omega_{-}(k)=$ $v_{-}|k|+O\left(k^{2}\right)$. The velocity of the linear mode, at the criticality is $v_{-}=g_{\gamma} / g$ and the transition belongs to the 2D Ising universality class [21]. For $g_{\gamma}=0$ (with additional $U$ (1) symmetry corresponding to conservation of chirality), at $q=q_{c}=g / 2$, the linear velocity vanishes and the Ising transition transforms into a commensurate-incommensurate one [20] with characteristic quadratic (nonrelativistic) lowenergy dispersion $\omega_{-}(k) \simeq k^{2} / 2 g$.

The dimer phase is realized for $q<q_{c}$ and transforms into singlet phases for $q>q_{c}$. Two singlet phases, corresponding to $g_{\gamma}>0 \rightarrow\left\langle\theta_{v}\right\rangle=0$ and $g_{\gamma}<0 \rightarrow\left\langle\theta_{v}\right\rangle=\sqrt{\pi} / 2$ cases, respectively, are separated by a Gaussian phase transition between each other at smaller values of $q>q_{c}$, however, both of them evolve adiabatically into the BI state with increasing $q$. The singlet phases can be approximated as $|S\rangle \sim \prod_{j}|S\rangle_{j}$, where $|S\rangle_{j}=\left|\frac{3}{2}\right\rangle_{j} \otimes\left|-\frac{3}{2}\right\rangle_{j}-\zeta_{ \pm}\left|\frac{1}{2}\right\rangle_{j} \otimes\left|-\frac{1}{2}\right\rangle_{j}$, with $\zeta_{ \pm} \rightarrow$ \pm 1 for $q \rightarrow 0$ and $\left|\zeta_{ \pm}\right| \rightarrow 0$ after the crossover into BI. The dependence of chirality and chirality susceptibility obtained from the effective model Eq. (2) at the dimer-to-singlet-phase transitions for different values of $g_{\gamma}$ is depicted in Fig. 2.

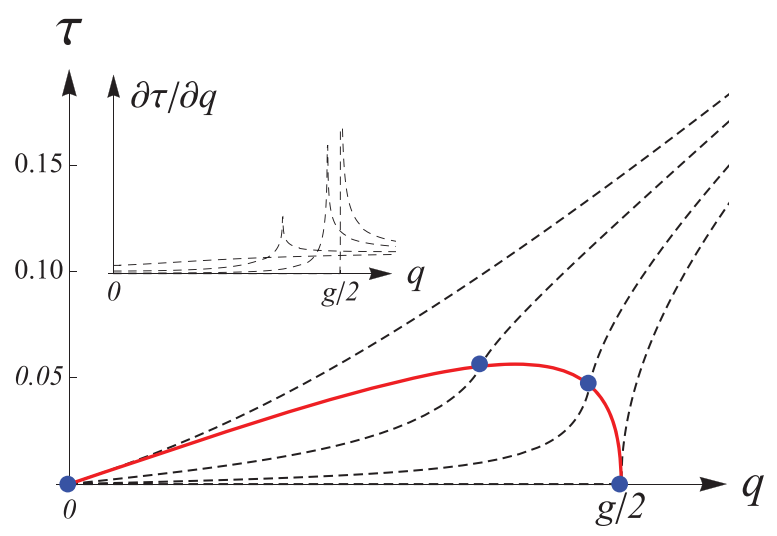

FIG. 2. (Color online) Chirality versus quadratic Zeeman coupling (dashed lines) for different values of $g_{\gamma}\left(g_{\gamma}=0\right.$ for the rightmost curve and $g_{\gamma}=g$ for the leftmost curve). Intersections of the continuous line with the dashed lines give $q_{c}$ and critical value of chirality. Inset shows chirality susceptibility and for rightmost curve the divergence corresponds to the commensurate-incommensurate phase transition. 

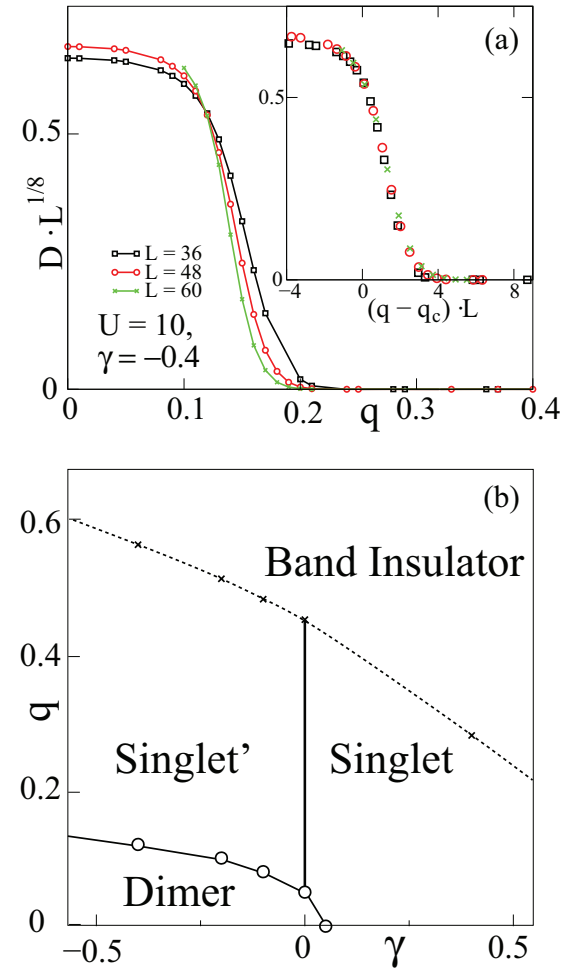

FIG. 3. (Color online) (a) Scaling of the dimer order parameter in the vicinity of dimer-to-singlet-phase transition for different system sizes. The inset shows the collapse of all finite-system-size data to a single curve, confirming Ising criticality. (b) Ground-state phase diagram for repulsive $f=3 / 2$ alkali-metal fermions at half-filling, as function of $q$ (in units of $t$ ) and $\gamma=\left(g_{2}-g_{0}\right) / 2 t$ for the case of $\left(g_{2}+g_{0}\right) / 2=10 t$.

To check the phase diagram at strong couplings (relevant for experiments) we use density matrix renormalization group (DMRG) calculations for open boundary conditions [22]. We keep on average up to 800 states and $L=60$ sites, comparable to the number of occupied lattice sites per chain in [23]. We monitor directly dimer order parameter $D$. The scaling of dimer order and a collapse of the data for different system sizes on a single curve depicted in Fig. 3(a) confirms the Ising character of dimer to singlet phase transition $D \sim$ $\Theta\left(q_{c}-q\right)\left(q_{c}-q\right)^{1 / 8}$ for $q \rightarrow q_{c}$ as predicted from effective model Eq. (2). The numerical ground-state phase diagram for $f=3 / 2$ fermions at half-filling is depicted in Fig. 3(b). Fidelity susceptibility (not shown) has a pronounced peak, both at dimer-to-singlet-phase transition as well as at the singlet to $\mathrm{BI}$ crossover, however, for the latter case the height does not scale with system size, indicating absence of phase transition, but rather the presence of a sharp crossover between singlet and BI states. In the BI state chirality (quasi)saturates $\tau \simeq 1$.

The Ising phase transition between dimer and singlet phases, induced by an external magnetic field, generalizes the one that was predicted for $q=0$ and $g_{\gamma}=g$ using bosonization analysis [19] when changing the scattering lengths $a_{0}$ or $a_{2}$. In case of attractively interacting $f=3 / 2$ fermions similar phase transition happens between singlet and quartetting phases [24,25]. Our work suggests that this phase transition can be induced by an external magnetic field via quadratic Zeeman coupling, without the need to change scattering lengths, thereby presenting certain advantages for experiments.

Relevant model for ${ }^{40} \mathrm{~K}$ atoms. To address the nature of ground states for the four-component mixture of ${ }^{40} \mathrm{~K}$ atoms we have to break the rotational symmetry associated with the hyperfine spin $f=3 / 2$. We relabel the states from the $f=9 / 2$ manifold that were used in experiment [23] as follows, $m_{f}=9 / 2 \rightarrow 3 / 2, m_{f}=1 / 2 \rightarrow-3 / 2, m_{f}=7 / 2 \rightarrow$ $1 / 2$, and $m_{f}=3 / 2 \rightarrow-1 / 2$, so that the lattice Hamiltonian is again given by Eq. (1), but with the modified quartic part,

$$
\begin{aligned}
H_{\text {int }, j}= & \sum_{\alpha<\beta} U_{\alpha, \beta} n_{\alpha, j} n_{\beta, j} \\
& +U_{\gamma}\left[\psi_{\frac{1}{2}, j}^{\dagger} \psi_{-\frac{1}{2}, j}^{\dagger} \psi_{\frac{3}{2}, j} \psi_{-\frac{3}{2}, j}+\text { H.c. }\right] .
\end{aligned}
$$

All interaction constants, $U_{\alpha, \beta}$, are different combinations of the scattering lengths $\left(a_{2}^{K}, a_{4}^{K}, a_{6}^{K}, a_{8}^{K}\right)$ reported in [23] and spin-changing interaction amplitude $U_{\gamma} \sim \frac{4 \pi \hbar^{2}}{m}\left(a_{8}^{K}-a_{6}^{K}\right)$.

For $q=0$ starting from the $t=0$ and performing degenerate perturbation theory in $t^{2} / U$ one can show that a classical (Ising) Néel state is energetically favorable in the deep lattice limit. The Néel state is made of the original $m_{f}=1 / 2$ and $m_{f}=3 / 2$ components paired on odd sites while $m_{f}=9 / 2$ and $m_{f}=7 / 2$ paired on even sites (or vice versa). The pairing of $m_{f}=1 / 2$ and $m_{f}=3 / 2$ components minimizes the interaction energy per lattice site, and due to constraint: $N_{1 / 2}=N_{9 / 2}$ and $N_{3 / 2}=N_{7 / 2}$, other $L / 2$ sites are occupied by $m_{f}=9 / 2$ and $m_{f}=7 / 2$ pairs. Activating $t$ introduces Néel order due to delocalization energy gain.

To study effects of quadratic Zeeman coupling we use infinite system size DMRG [26] calculations, keeping on average 200 states, and monitor different order parameters depicted in Fig. 4. Explicit expressions of nonlocal parity [27] and string orders can be borrowed from the corresponding orders of $S=1$ spin chain [28] by identifying Néel up ( $m_{f}=1 / 2$ and $m_{f}=3 / 2$ pairs) and down $\left(m_{f}=9 / 2\right.$ and $m_{f}=7 / 2$ pairs) components with $S^{z}=+1$ and $S^{z}=-1$ states, $S_{j}^{Z}=\left(n_{\frac{9}{2}, j}+n_{\frac{7}{2}, j}-n_{\frac{3}{2}, j}-n_{\frac{1}{2}, j}\right) / 2$ and on-site singlets (initial pairs of BI composed of $m_{f}=9 / 2$ and $m_{f}=1 / 2$

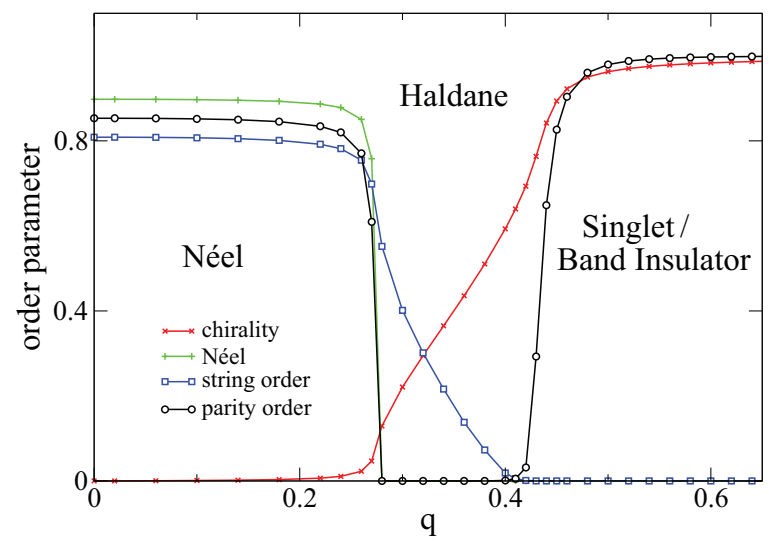

FIG. 4. (Color online) Quadratic Zeeman coupling (measured in units of $t$ ) dependence of the order parameters along a cut of Fig. 1 for $U / t=20$. Neel, parity, and string orders are given, respectively, by $\lim _{n \gg 1}(-1)^{n}\left\langle S_{j}^{z} S_{j+n}^{z}\right\rangle,\left\langle e^{i \pi \sum_{j} S_{j}^{z}}\right\rangle$, and $\lim _{n \gg 1}\left\langle S_{j}^{z} e^{i \pi \sum_{j<k<j+n} S_{k}^{z}} S_{j+n}^{z}\right\rangle$. 
components) with $S^{z}=0$. The sequences of phase transitions in strong coupling induced by $q$ are shown in Fig. 4. For deep lattices the emergence of Néel and Haldane phases in the presence of quadratic Zeeman coupling is a generic feature of four-component fermions with spin-changing processes allowed for $f \neq 3 / 2$. The Haldane phase can intuitively be understood as a Néel order diluted with the defects that represent on-site singlets of the BI state.

For shallow lattices, as depicted in Fig. 1, a dimer state wins, consistent with weak-coupling bosonization analysis. For the experimental values of scattering lengths in [23], the transition from dimer to Néel state happens in the strong coupling regime with increasing lattice depth, where bosonization cannot be trusted. However one can induce similar phase transition in weak coupling by changing interaction coefficients in Eq. (3). In the dimer phase the fields are pinned as follows: $\left\langle\phi_{v}\right\rangle=$ $\left\langle\phi_{t 1}\right\rangle=\left\langle\phi_{t 2}\right\rangle=0$, whereas in the Néel phase, $\left\langle\phi_{v}\right\rangle=\left\langle\phi_{t 1}\right\rangle=$ 0 and $\left\langle\phi_{t 2}\right\rangle=\sqrt{\pi} / 2$ [19]. Hence, in dimer-to-Néel transition only the $\phi_{t_{2}}$ sector is involved, and moreover one can show that the phase transition is Gaussian. With increasing $q$ an Ising phase transition happens from Néel to Haldane phase in the $\phi_{v}$ sector also captured by the effective model of Eq. (2). The expectation values of bosonic fields in the Haldane phase are as follows: $\left\langle\theta_{v}\right\rangle=\left\langle\phi_{t 1}\right\rangle=0$ and $\left\langle\phi_{t 2}\right\rangle=\sqrt{\pi} / 2$, and with further increasing $q$ the singlet state should be recovered, with $\left\langle\theta_{v}\right\rangle=$ $\left\langle\phi_{t 1}\right\rangle=\left\langle\phi_{t 2}\right\rangle=0$. Occurrence of the Haldane phase between Néel and singlet MI is thus natural in bosonization. Between the Haldane phase and singlet (like between dimer and Néel) a Gaussian criticality from the gapless $\phi_{t_{2}}$ sector is expected, characterized by nonuniversal exponents. This is in agreement with DMRG results failing to capture universal exponents at these phase transitions and confirming their smooth nature (second order). The complete numerical ground-state diagram is presented in Fig. 1.

In summary. A rich ground-state phase diagram of halffilled four-component repulsively interacting alkali-metal fermions can be explored by changing the lattice depth and the quadratic Zeeman coupling, without the need to change the scattering lengths. For the case of $f=3 / 2$ fermions, dimer and singlet phases are realized; whereas when four components are from $f>3 / 2$ multiplet, like ${ }^{40} \mathrm{~K}$ atoms, four distinct gapped MI phases are expected. The primary candidate to observe different MI states presented in this work is a system of ${ }^{40} \mathrm{~K}$ atoms under the similar conditions as in recent experiment [23] where initially a state of two fermions per site was stabilized by the quadratic Zeeman effect, the starting state being a BI of the two-component Fermi Hubbard model. Although the ${ }^{40} \mathrm{~K}$ atoms were loaded in a $3 \mathrm{D}$ lattice, hopping was allowed only along one direction, and since $s$-wave interactions are felt on-site the system studied experimentally was a collection of $1 \mathrm{D}$ decoupled lattices. If the spin-changing processes will be activated by adiabatically reducing the magnetic field, different MI states of Fig. 1 may be reached.

These MI phases may be detected with the current state-ofthe-art techniques, e.g., using Faraday rotation as proposed in [29]. Due to a shallow harmonic trap along the lattice the MI phases will occupy the same central region as the BI does [23]. For the case $f \neq 3 / 2$ we have neglected the finite possibility of production of other than four hyperfine components. Indeed for deep lattices the number of $m_{f}=$ $5 / 2$ and $m_{f}=-1 / 2$ components produced after the quench of magnetic field in the BI state is negligibly small [23]. However, one can use this possibility to pinpoint the Néel phase using Stern-Gerlach techniques in time of flight. If one freezes the ground-state configuration by sudden increase of lattice depth only $m_{f}=1 / 2$ and $m_{f}=3 / 2$ pairs can produce $m_{f}=5 / 2$ and $m_{f}=-1 / 2$ components and this process will be maximally enhanced in the Néel phase, whereas $m_{f}=9 / 2$ and $m_{f}=7 / 2$ components do not experience spin-changing collisions, hence their number will not decrease in time.

This work has been supported by Center for Quantum Engineering and Space-Time Research (QUEST) and DFG Research Training Group (Graduiertenkolleg) 1729.
[1] M. Lewenstein, A. Sanpera, V. Ahufinger, B. Damski, A. S. De, and U. Sen, Adv. Phys. 56, 243 (2007).

[2] I. Bloch, J. Dalibard, and W. Zwerger, Rev. Mod. Phys. 80, 885 (2008).

[3] R. Jördens, N. Strohmaier, K. Günter, H. Moritz, and T. Esslinger, Nature (London) 455, 204 (2008).

[4] U. Schneider et al., Science 322, 1520 (2008).

[5] R. Jördens et al., Phys. Rev. Lett. 104, 180401 (2010).

[6] D. McKay and B. DeMarco, Rep. Prog. Phys. 74, 054401 (2011).

[7] M. Hermele, V. Gurarie, and A. M. Rey, Phys. Rev. Lett. 103, 135301 (2009).

[8] E. Szirmai and M. Lewenstein, Europhys. Lett. 93, 66005 (2011).

[9] H. Nonne, P. Lecheminant, S. Capponi, G. Roux, and E. Boulat, Phys. Rev. B 84, 125123 (2011).

[10] H. Nonne, M. Moliner, S. Capponi, P. Lecheminant, and K. Totsuka, Europhys. Lett. 102, 37008 (2013).
[11] Considering optical lattice constant of 0.5 microns and keeping lattice depth fixed at $35 E_{R}$ in two (perpendicular to the chain) directions, parameter span $6<U / t<30$ corresponds to lattice depths roughly between $4 E_{R}$ and $10 E_{R}$, where $E_{R}$ is the recoil energy. This is well within the experimental range of [23].

[12] C. Wu, J. P. Hu, and S. C. Zhang, Phys. Rev. Lett. 91, 186402 (2003).

[13] K. Rodriguez, A. Argüelles, M. Colome-Tatché, T. Vekua, and L. Santos, Phys. Rev. Lett. 105, 050402 (2010).

[14] Hyperfine spin $f=3 / 2$ have ${ }^{9} \mathrm{Be},{ }^{132} \mathrm{Cs}$, and ${ }^{201} \mathrm{Hg}$ atoms.

[15] D. Jaksch, C. Bruder, J. I. Cirac, C. W. Gardiner, and P. Zoller, Phys. Rev. Lett. 81, 3108 (1998).

[16] J. B. Marston and I. Affleck, Phys. Rev. B 39, 11538 (1989).

[17] K. Buchta, Ö. Legeza, E. Szirmai, and J. Sólyom, Phys. Rev. B 75, 155108 (2007). 
[18] A. V. Gorshkov et al., Nature Physics 6, 289 (2010).

[19] C. Wu, Mod. Phys. Lett. B 20, 1707 (2006).

[20] A. O. Gogolin, A. A. Nersesyan, and A. M. Tsvelik, Bosonization and Strongly Correlated Systems (Cambridge University Press, Cambridge, 1998).

[21] Y.-J. Wang, arXiv:cond-mat/0306365; F. H. L. Essler and I. Affleck, J. Stat. Mech. (2004) P12006.

[22] S. R. White, Phys. Rev. Lett. 69, 2863 (1992); Phys. Rev. B 48, 10345 (1993).

[23] J. S. Krauser et al., Nature Phys. 8, 813 (2012).
[24] P. Lecheminant, E. Boulat, and P. Azaria, Phys. Rev. Lett. 95, 240402 (2005); G. Roux, S. Capponi, P. Lecheminant, and P. Azaria, Eur. Phys. J. B 68, 293 (2008).

[25] C. Wu, Phys. Rev. Lett. 95, 266404 (2005).

[26] U. Schollwöck, Rev. Mod. Phys. 77, 259 (2005).

[27] S. P. Rath, W. Simeth, M. Endres, and W. Zwerger, Ann. Phys. (NY) 334, 256 (2013).

[28] S. Greschner, L. Santos, and T. Vekua, Phys. Rev. A 87, 033609 (2013).

[29] K. Eckert et al., Nature Phys. 4, 50 (2007). 